\title{
Zwischen EEG und EGG
}

\section{Eve Stockhammer}

Dr. med., Psychiaterin und Psychotherapeutin

Als ich in den 90er Jahren meine neurophysiologische Ausbildung in der Schweizerischen Epilepsie-Klinik (EPI) absolvierte, just in der Zeit der digitalen Wende, packte mich das «EEG-Fieber»: Im dunklen Untergeschoss der EPI traf ich auf eine kleine verschworene Gruppe von angefressenen EEG-isten, Schlaf-Forscherinnen und -Forschern, die mich lehrten, durch die Partitur des oberflächlichen Hirnstrombildes (vorerst auf Papier, dann digitalisiert) in vielseitige neurophysiologische Tiefen zu blicken.

Es war ein bisschen wie Musik! Das geübte Auge erkannte im Kurvenbild die Dysfunktionen wie ein musikalisches Ohr die Dissonanzen; dies führte zur Passion! So schwer die Krankheitsbilder und ernst die Diagnostik, es herrschte stets eine Stimmung von Faszination und Begeisterung im EEG-Labor, eine Art von Forscherstimmung, als würde über uns noch ein bisschen der Geist unseres hochverehrten geistigen Mentors schweben, des EEG-Entdeckers Hans Berger.

Ja, auf diesen Hans Berger waren wir mächtig stolz, und seine bis dahin bekannte Biographie bot ja auch allen Grund dazu, ihn zu verehren und ihm - im stillen Kämmerlein - nachzueifern.

2012, nach einer fast 20-jährigen Latenzzeit, begann ich mich erneut mit Hans Berger zu beschäftigen, ausgelöst durch eine Buchbesprechungs-Anfrage. Dabei

$\rightarrow$ Aktuelle Ausgabe ode

$\rightarrow$ Archiv $\rightarrow 2016 \rightarrow 39$

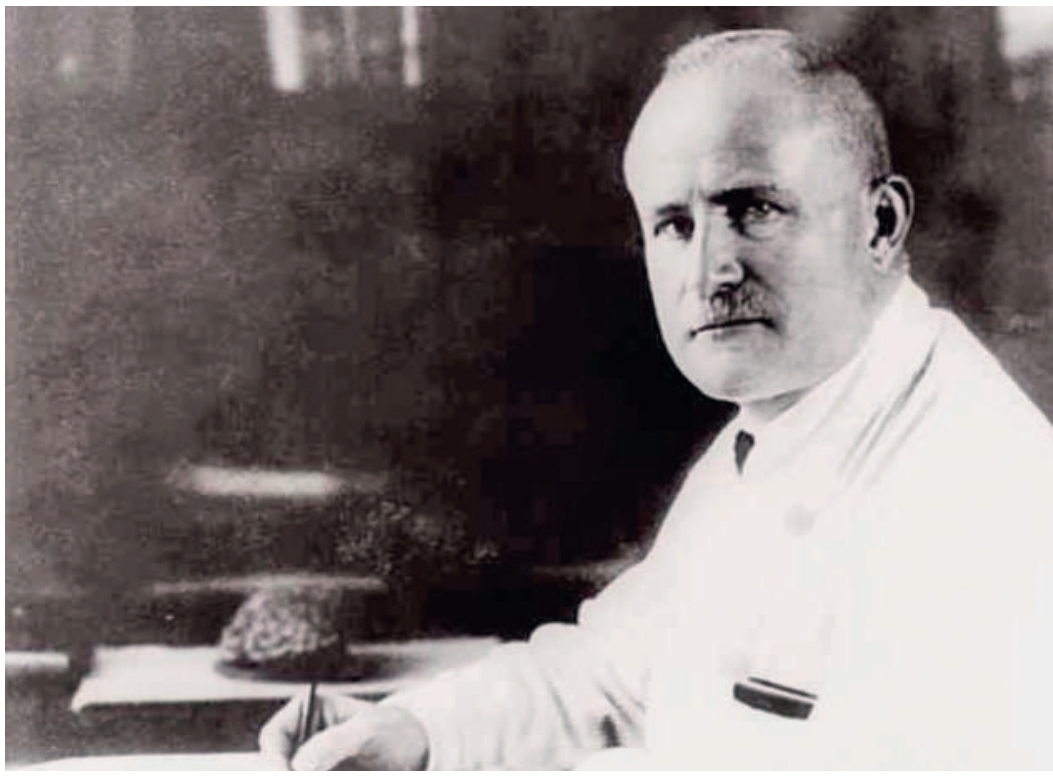

Hans Berger (1873-1941), Entdecker der Elektroenzephalographie (EEG). wurde ich mit neuen medizinhistorischen Facts über den EEG-Entdecker konfrontiert. Nach dem Tod der Witfrau Berger konnte erstmals sein umfänglicher Nachlass inklusive Tagebücher der medizinhistorischen Forschung zugänglich gemacht werden. In ausführlichen Publikationen von Susanne Zimmermann und Uwe Hossfeld über die Verstrickungen der Uni Jena in den Nationalsozialismus erfuhr ich nun, dass Hans Berger nicht nur «förderndes Mitglied» der SS war und antisemitische Passagen in seine Tagebücher schrieb, sondern dass er seine rassenbiologischen Überzeugungen auch in die Tat umgesetzt hatte:

\section{Diese Tatsachen liessen mich nicht kalt.}

Berger war in den dreissiger Jahren freiwillig als Gutachter für das Erbgesundheitsgericht (EGG) und das Erbgesundheitsobergericht (EGOG) tätig und damit direkt dafür verantwortlich, dass viele ihm anvertraute Patienten, darunter eine grosse Anzahl von Epileptikern und Psychiatriepatienten, zwangssterilisiert wurden.

Diese Tatsachen liessen mich nicht kalt. Warum hatte sich der Wissenschaftler Hans Berger so aktiv für die Zwangssterilisation eingesetzt? Als Klinikleiter, Dekan und späterer Universitätsrektor dürfte er wohl kaum gegen seine Überzeugung dazu gezwungen worden sein. Die neuere medizinhistorische Literatur hilft hier weiter. Sie lässt die Leserin, den Leser in die philosophische und naturwissenschaftliche Vorstellungswelt des beginnenden Zwanzigsten Jahrhunderts eintauchen. Und sie ermöglicht, das Denken der geistigen Wegbereiter für die Nazi-Ideologie besser nachvollziehen zu können, die wenige Jahre später an der Universität Jena so unbeschwert ihre Blüten treiben sollte.

\section{Die wissenschaftliche Geisteswelt von Hans Berger}

Der Arztsohn Hans Berger, 1873 im heutigen Coburg geboren, interessierte sich bereits in jungen Jahren für das Grenzgebiet zwischen Neurologie und Psychiatrie, ausgelöst durch ein persönliches Kriegserlebnis, das er als telepathische Übertragung interpretierte. Tief beindruckt von diesem Vorkommnis machte er es sich zum Ziel, die sogenannte "psychische Energie» mess- 
bar und physisch erfassbar zu machen. Die jahrzehntelange Suche danach, beginnend bei Messungen der Hirntemperatur sowie Aufzeichnungen der Hirnzirkulation, führte schlussendlich, über die Anwendung von Schädelelektroden, zur Entdeckung des humanen EEGs.

\section{Warum hatte sich der Wissenschaftler Hans Berger so aktiv für die Zwangssterilisation eingesetzt?}

Die Basis für Bergers philosophisch-wissenschaftliche Überzeugungen bildete die wissenschaftliche Geisteswelt des späten 19. Jahrhunderts. Grundlegend dabei war der von den meisten damaligen Wissenschaftlern geteilte reduktionistische Glaube an die direkte Messbarkeit einer materiellen Hirnspur, sei sie anatomisch, physiologisch oder chemisch, als erfassbarer Ausdruck jeglicher psychischer Vorgänge, inklusive Intelligenz und Psychopathologie. Es war die Blütezeit der Eugeniker und Monisten, die ausgehend von den Naturwissenschaften jeglichen Dualismus von Geist/Materie und Gott/Natur ablehnten und die sich 1906 unter der Führung des Biologen und "Antipapstes» Ernst Haeckel mit dem Physikochemiker Wilhelm Ostwald an der Universität Jena zum Deutschen Monistenbund zusammenschlossen. Sie alle waren geistige Väter von Hans Berger. Viele Anhänger dieser vorerst freigeistigen Gemeinschaft von «Wissenschafts- und Fortschrittsgläubigen» wurden in den Folgejahren durch Verdichtung von Elitebewusstsein, Züchtungsgedanken und radikaler Eugenik zu geistigen und praktischen Wegbereitern der Zwangssterilisation und selbst der Euthanasie.

\section{Umgang mit Schutzbefohlenen}

Hans Berger war zweifellos ein passionierter und hervorragender Hirnforscher, der jahrelang akribisch seine Untersuchungsinstrumente verbesserte, fleissig Tage und Nächte lang Ableitungen durchführte und bahnbrechende Forschungsresultate vorlegte. Patienten hingegen interessierten ihn, glaubt man seinen Schülern, vornehmlich als Forschungsmaterial. Wie auch in seinen Publikationen zu lesen ist, liess er beispielsweise seine «Idioten" stundenlang warm baden, um das Widerstreben dieser Kranken zu brechen, ohne ihnen Beruhigungsmittel geben zu müssen, da diese sonst das EEG verfälscht hätten. Dass die Rechte und Interessen von Patienten in der Forschung kaum eine Bedeutung hatten, war keine Spezialität Bergers, sondern fast schon «normal» für Psychiater in dieser Zeit. Mit unseren heutigen Massstäben sehen wir darin einen Patientenmissbrauch. Geisteskrankheiten wurden damals von den meisten Psychiatern als eine Folgeerscheinung von Vererbung und Degeneration verstanden, für Patientengespräche gab es wenig Platz. Von Berger wurden sie als «Zeitverschwendung» eingestuft, wie es ein ehemaliger Schüler einschätzte. Mit eben dieser Einstellung gegenüber Patienten, gepaart mit rassenbiologischen Grundüberzeugungen, war dann der Schritt zur Gutachtertätigkeit für die Durchführung der Zwangssterilisation nicht mehr weit, für Hans Berger schon fast eine «logische» Folge.

In Bergers wissenschaftlicher und moralischer Geisteswelt stellte also seine EEG-Labortätigkeit für die Epilepsieforschung und sein EGG-Engagement gegen die Fruchtbarkeit der Epileptiker keinen Widerspruch dar. Er verschloss sich konsequent anderen philosophischen und psychologischen Auffassungen. So bewertete er beispielsweise die Schriften von Sigmund Freud als einen «Rückfall in primitive Zustände». An dieser radikal materialistischen Vorstellung von einer «Psychologie ohne Seele», die jegliche Psychopathologie im Neuron der Hirnrinde sucht und das Unbewusste strikt ablehnt, hielt Berger unbeirrt sein ganzes Leben lang fest. Ein Leben, das dann auch, offenbar in einer schweren depressiven Krise, durch Erhängen im Südtrakt der Psychiatrischen Klinik Jena ein Ende fand.

\section{Widersprüche aushalten}

75 Jahre nach seinem Tod ist die Wahrnehmung von Hans Berger immer noch gespalten: Sowohl eine Jenaer Strasse, die dortige Neurologische Universitätsklinik wie auch ein hochangesehener Preis tragen seinen Namen. In gewissen eher repräsentativen Publikationen wird Hans Berger ausschliesslich für seine Verdienste geehrt, während in anderen, vor allem historischen Veröffentlichungen, seine Gesamtpersönlichkeit unter Einbezug der Zwangssterilisations-Problematik dargestellt wird.

Es ist unbequem, wenn bei einem verehrten Wissenschaftler Mittätertum in der Nazizeit bekannt wird; bedenklich wird es, wenn die Antwort darauf nur aus Abwehr besteht. Alternativ sollten Widersprüche ausgehalten und Heroengeschichten, wie die von Hans Berger, hinterfragt werden.

Dabei stellen sich auch Fragen an die medizinischen Fachgesellschaften, wie mit einem entsprechenden Preis- respektive Kliniknamen künftig umgegangen werden soll; Fragen, die vielleicht am fruchtbarsten interdisziplinär, unter Beihilfe von spezialisierten Historikern, diskutiert werden könnten.

\section{Bildnachweis}

https://de.wikipedia.org/wiki/Hans Berger \%28Neurologe\%29\#/ media/File:HansBerger_Univ_Jena.jpeg 


\section{Literatur}

- Zimmermann S. Die Medizinische Fakultät der Universität Jena während der Zeit des Nationalsozialismus. Berlin: VBW-Verlag; 2000. S. 146

- Fields D. The other brain. New York: Simon \& Schuster; 2009. S. 150

- Hossfeld U, John J, Lemuth O, Stutz R. Kämpferische Wissenschaft. Köln: Böhlau; 2003. S. 314.

- Demmler A. Wilhelm Strohmayer (1874-1936). Ein Wegbereiter der Kinder- und Jugendpsychiatrie. Dissertation (Universität Jena 2003). S. 29, 40.

- Klee E. Deutsche Medizin im Dritten Reich. Frankfurt am Main Fischer; 2001. S. 78-81.

- Berger H. Das Elektroenkephalogramm des Menschen. Nova Acta Leopoldina. 1938;6:291-2.
- Zeidman L, Stone J, Kondziella D. New revelations about Hans Berger, father of the electroencephalogram (EEG), and his ties to the Third Reich. J. of Child Neurology. 2014;29:1002-10.

- Duncker T. Energie und Bewusstsein. Untersuchungen zur Psychophysiologie Hans Bergers. Dissertation (Marburg 2004).

- Borck C. Hirnströme, eine Kulturgeschichte der Elektroenzephalographie. Göttingen: Wallstein; 2005.

- Bauer J, Kluge H. Das wissenschaftliche Gesamtwerk des Jenaer Nervenarztes Hans Berger. Stuttgart: Steiner; 2011.

- Westermann S. Verschwiegenes Leid, der Umgang mit den NSZwangssterilisationen in der Bundesrepublik Deutschland. Köln Böhlau; 2010 